\title{
Die Kornblume - Lieblingsblume der Preußen
}

\author{
Peter Schubert \& Hilke Steinecke
}

\begin{abstract}
Cornflower (Centaurea cyanus) is a well known weed in fields and of great symbol value. It was a favourite plant of the Prussian Queen Luise and her son, Wilhelm I. Biology, mythology and symbolic features of the cornflower are outlined.
\end{abstract}

\section{Zusammenfassung}

Die Kornblume (Centaurea cyanus) ist ein bekanntes Ackerwildkraut. Sie hat aber auch großen Symbolwert und gilt z. B. als die Lieblingspflanze der Preußenkönigin Luise und ihres Sohnes, Kaiser Wilhelm I. Biologie, Mythologie und Symbolik werden vorgestellt.

\section{Attraktives Ackerwildkraut}

Im Gegensatz zu vielen anderen heimischen Wildblumen erfreut sich die Kornblume (Centaurea cyanus, Asteraceae) einer großen Bekanntheit. Kommt sie doch fast überall in Deutschland in Getreideäckern vor, auch wenn sie aufgrund des hohen Herbizideinsatzes, besserer Saatgutreinigung und Überdüngung seit einigen Jahren stellenweise leider recht selten geworden ist. Anderseits haben sich die Populationen dort, wo biologischer Landbau betrieben wird oder Ackerrandstreifen nicht gespritzt werden, wieder etwas erholt. Auf Äckern oder Brachland ist die Kornblume oft in Gesellschaft von Klatschmohn oder Kamille anzutreffen.

Abb. 1: Acker mit bunt blühenden Wildkräutern, darunter Kornblume und Klatschmohn.

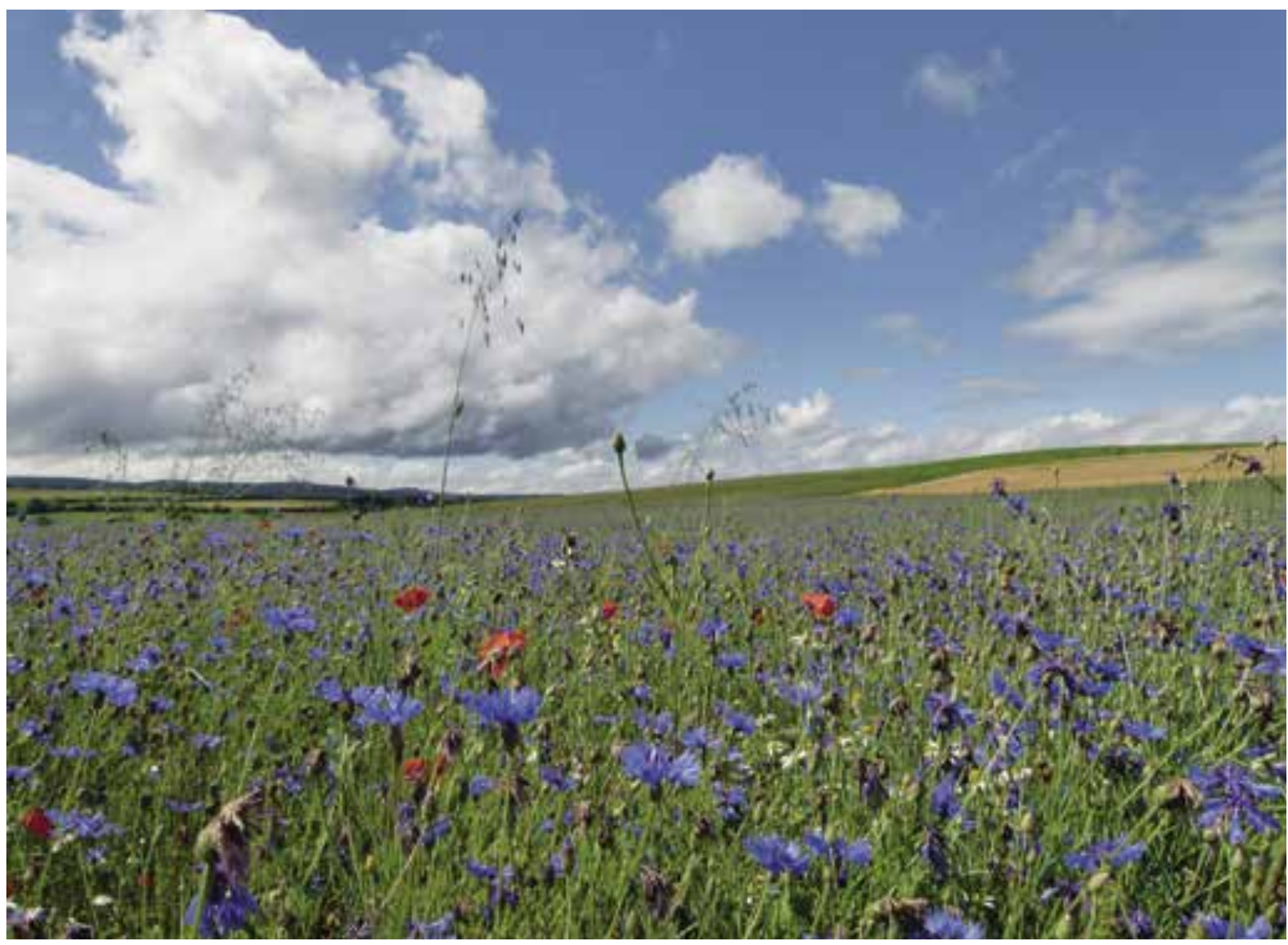


Die Kornblume ist ein ein- bis zweijähriger krautiger Vertreter der Korbblütler. Kornblumen überwintern einerseits als Samen und keimen im Frühling aus. Anderseits können die Samen bereits im Herbst keimen, dann überwintern die Jungpflanzen. Kornblumen gedeihen besonders gut auf durchlässigen Böden an sonnigen Standorten. Die verzweigte, über einen halben Meter hohe Kornblume hat mehr oder weniger ungeteilte Blätter, die auf der Unterseite stark behaart sind. Vom Sommer bis in den Oktober hinein bilden sich die blauen Blütenköpfchen, die wie eine Einzelblüte wirken. Das Köpfchen enthält nur Röhrenblüten Die äußeren Röhrenblüten sind steril, blütenblattartig vergrößert und übernehmen die Schaufunktion. Neben der blauen Wildform gibt es auch Sorten mit weißen oder rosa gefärbten Blüten.

Kornblumen werden gelegentlich an Straßenböschungen oder Wegrändern ausgesät. Sie sind auch häufig in einjährigen Wildblumenbzw. Insektenblumenmischungen vorhanden. Im Garten eignen sich Kornblumen dafür, Freiflächen schnell mit einem sommerlichen Blütenflor zu überziehen und dabei gleichzeitig Futterpflanzen für Hummeln, Bienen oder Schmetterlinge anzubieten. Die Samen können etwa im April direkt auf der entsprechenden Fläche ausgesät werden. Eng verwandt mit der Kornblume sind die Flockenblumen, ebenfalls Vertreter der Gattung Centaurea. Flockenblumen (z. B. die heimische Skabiosen-Flockenblume, Centaurea scabiosa) sind mehrjährig und gut geeignet für das Anlegen naturnaher Gärten und dauerhafter Magerwiesen.

\section{Centaurea in der griechischen Mythologie}

Die Kornblume ist ursprünglich nicht in Mitteleuropa heimisch gewesen. Sie kam mit dem Getreideanbau vermutlich aus dem östlichen Mittelmeergebiet und Vorderasien zu uns. Bereits bei den alten Griechen wurde für sie die Bezeichnung Centaurea verwendet. CARL v. LINNÉ griff diesen Namen auf und wählte für die Kornblume 1753 den wissenschaftlichen Namen Centaurea cyanus. Der Artbeiname cyanus leitet sich von der griechischen Vokabel kyanos für blau ab. Der Gattungsname bezieht sich auf kentauros = Kentaur, ein Mischwesen aus Pferd und Mensch. Chiron (oder auch Cheiron), einer der berühmtesten Kentauren aus der griechischen Mythologie, war ein Halbbruder des Zeus. Er soll nach griechischer Sage der Heilkunst kundig gewesen sein und brachte dem Götterarzt Asclepios medizinisches Wissen bei. Cheiron lebte in einer Höhle am Fuße des Berges Pelion (Pilion) in Thessalien, der namensgebend für die gesamte Halbinsel Pilion ist. Es handelt sich um einen vom Tourismus noch ziemlich umberührten und traumhaft schönen Flecken auf griechischem Festland. In einem Streit wurde Cheiron von einem Pfeil verletzt, der von Herkules abgeschossen und mit dem Gift der Hydra präpariert worden war. Mit Hilfe der Kornblume linderte Cheiron die Schmerzen seiner Wunde (Baumann 1993).

\section{Kornblume als Heilpflanze}

Heute fällt der Kornblume medizinisch keine bedeutende Rolle mehr zu, früher jedoch wurden die getrockneten Blüten (Flores Cyani) tatsächlich zum Heilen genutzt. Die Blüten wurden z. B. als harntreibendes Mittel, als Augenwasser oder als Räucherpulver genutzt. Getrocknete Kornblumen-Blüten sind heute häufig (wohl eher aus optischen Gründen) diversen Kräutertees beigemischt, frische Kornblumenblüten sind essbar und können in Salaten farbliche Akzente setzen (Schönfelder \& SchöNFELDER 2001).

Im Kräuterbuch von LeOnHart Fuchs (1543) heißt es über die Blaw Kornblumen:

"Blüet im Brachmonat unnd Hewmonat am fürnemlichsten / ist gantz lieblich anzusehen ... / Diß kraut ist kalter natur unn treibt hindersich / wie man sölchs klärlich mag auß dem geschmack abnemen ... / Dise blaw Kornblum ist fürtreffenlich gut zu den hitzigen roten augen / und allen andern hitzigen gebresten in der erste / zerstossen und übergeschlagen. Sie ist auch nützlich zu bösen faulen wunden und schäden / zerstossen und den außgetruckten safft darinn gethon. Oder gedörrt unnd zu pulver gemacht/ unnd darinn gestrewet."

Ritter von Perger berichtet in seinen Deutschen Pflanzensagen von 1864: „Eine 


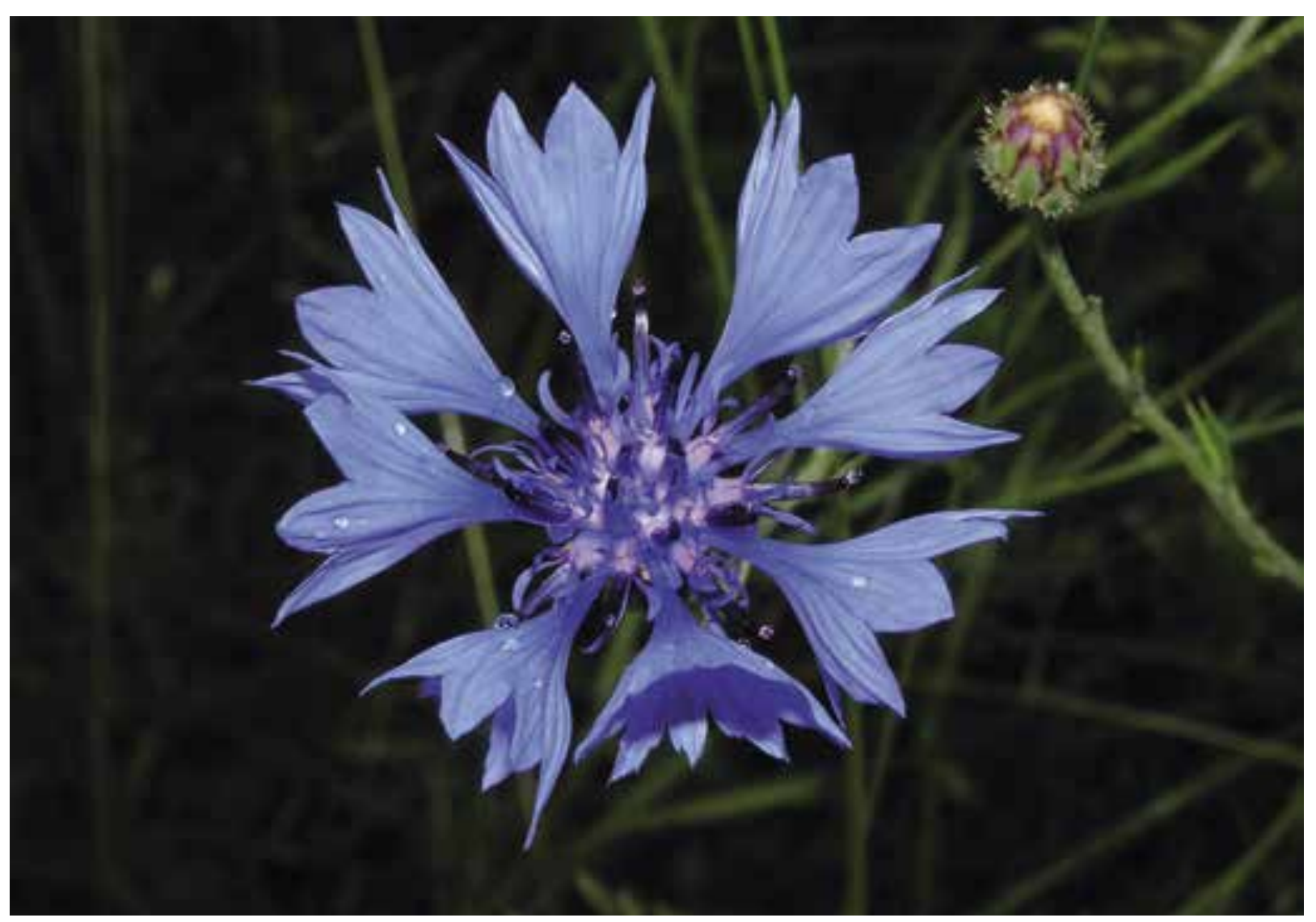

Kornblume am Fronleichnamstage ausgegraben, stillt das Nasenbluten, wenn man sie so lange in der Hand hält, bis sie erwärmt ist."

\section{Blume der Romantik}

Außer in der Sagenwelt oder in älteren Kräuterbüchern wurde über lange Zeit der Kornblume keine besondere Aufmerksamkeit gewidmet; sie wurde eher als ein lästiges Ackerunkraut betrachtet. Um 1800 stieg ihre Wertschätzung, denn die hübsche Wildblume mit den so auffällig blauen Blütenköpfchen sollte nun Einfachheit und Natürlichkeit verkörpern - Attribute, die dem damaligen Zeitgeist von Romantik und Aufklärung entsprachen. In der Literatur mag die blaue Blütenfarbe der Kornblume manch einen Poeten inspiriert haben. Literaten haben diskutiert, ob neben dem von Novalis selbst erwähnten Heliotrop möglicherweise die himmelblaue Wegwarte oder vielleicht die Kornblume Vorbilder für die berühmte blaue

Abb. 2: Blütenköpfchen einer Kornblume.
Blume gewesen sein könnten. Im 1800 entstandenen unvollendeten Romanfragment von Novalis" „Heinrich von Ofterdingen“ heißt es:

„Was ihn aber mit voller Macht anzog, war eine hohe lichtblaue Blume, die zunächst an der Quelle stand und ihn mit ihren breiten, glänzenden Blättern berührte. Rund um sie her standen unzählige Blumen von allen Farben, und der köstliche Geruch erfüllte die Luft. Er sah nichts als die blaue Blume und betrachtete sie lange mit unnennbarer Zärtlichkeit. Endlich wollte er sich ihr nähern, als sie auf einmal sich zu bewegen und zu verändern anfing; die Blätter wurden glänzender und schmiegten sich an den wachsenden Stengel, die Blume neigte sich nach ihm zu, und die Blütenblätter zeigten einen blauen ausgebreiteten Kragen, in welchem ein zartes Gesicht schwebte. Sein süßes Staunen wuchs mit der sonderbaren Verwandlung."

\section{Blume des Kaisers und der Königin}

Die Kornblume entwickelte sich zur Lieblingsblume von Persönlichkeiten, die einem recht fortschrittlichen, aufgeklärten und veränderten 


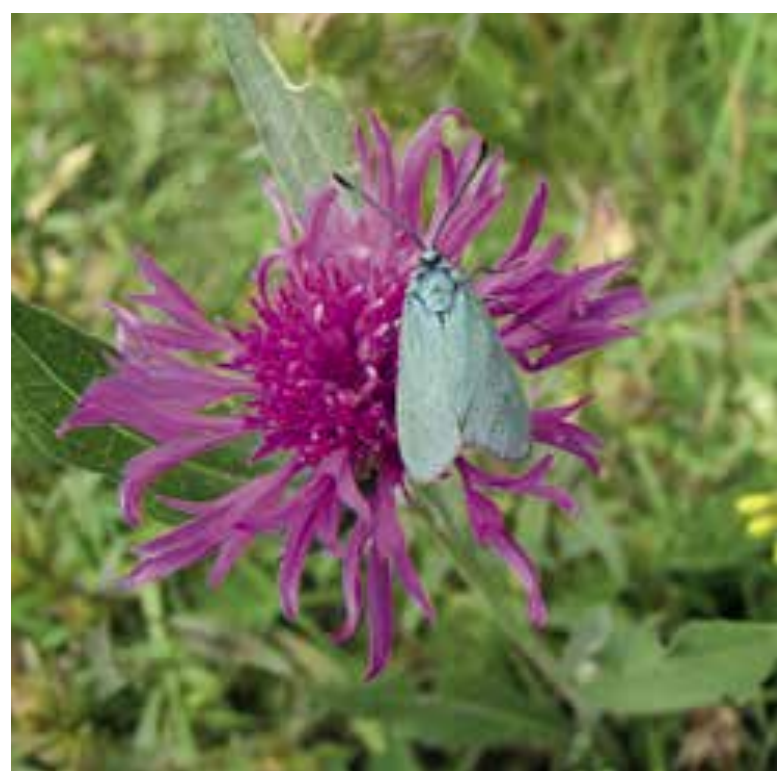

Lebensstil nachgingen. Dazu passte möglicherweise auch, dass die Kornblume als ein Zeichen der Veränderlichkeit galt, weil ihre Blütenfarbe mit zunehmendem Alter der Blüten ausbleicht.

Eine besondere Kornblumen-Geschichte rankt sich um Prinzessin Luise von Mecklenburg-Strelitz (1776-1810). Sie heiratete blutjung mit 17 Jahren den Kronprinzen FrIedRICH Wilhelm III. Nur wenige Jahre später wurde dieser im Alter von 27 Jahren zum König von Preußen gekrönt. LuIsE wurde somit, gerade mal 21 Jahre jung, Königin von Preußen. Aufgrund ihrer jugendlichen Schönheit, Herzlichkeit, unkomplizierten Umgangsformen und bürgerlichen Tugenden wurde sie vom Volk von Beginn ihrer Amtszeit an sehr geachtet. Ihr kam zugute, dass sie sich zusammen mit ihrem Gatten unter ganz normale Bürger mischte und sich auf Volksfesten oder auf dem Berliner Weihnachtsmarkt ohne Gefolge blicken ließ. Ganz öffentlich bekannten sich Luise und Friedrich Wilhelm als Liebespaar. 1808 berichtete Königin LuIsE über ihre gegenseitige Liebe:

„Er gefällt mir in allen Stücken und ich gefalle ihm und uns ist am wohlsten, wenn wir zusammen sind."

Aus der glücklichen Beziehung gingen 10 Kinder hervor, von denen nur drei früh im Kindesalter starben. Für die damalige Zeit eher ungewöhnlich und fortschrittlich kümmerte sich
LuISE persönlich um die Erziehung ihrer Kinder. Eltern und Kinder duzten sich, obwohl damals noch in der besseren Gesellschaft die gegenseitige Anrede mit "Sie“ üblich war. In Zeiten der Revolutionswirren und des Angriffs Napoleons kam es 1806 zur Niederlage Preußens in der Schlacht von Jena und Auerstedt. LuISE musste mit ihren Kindern nach Ostpreußen fliehen. Mit dabei war auch ihr zweitältester Sohn, der damals erst neunjährige Wilhelm, der spätere Kaiser Wilhelm I. Bei einem Unfall ging ein Rad der Kutsche entzwei, sodass am Feldrand eine Zwangspause eingelegt werden musste. Luise vertrieb sich und ihrer Familie die Zeit damit, ihren Kindern Kränze aus Kornblumen zu flechten. Diese Begebenheit muss den kleinen WILHELM sehr beeindruckt haben, denn er behielt sie bis ins Erwachsenenalter fest im Gedächtnis. Er deklarierte die einfache Kornblume zur Lieblingsblume seiner Mutter, passend zu ihrem bürgerlich-ungezwungenen Lebensstil. Als 1871 frisch ernannter Kaiser stellte Wilhelm, bevor er in den Krieg zog, einen Kornblumenstrauß auf das Grab seiner viel zu jung verstorbenen Mutter. Auch er machte die Kornblume zu seiner Lieblingsblume - vielleicht auch weil sie so schön „kornblumenblau“ bzw. „preußischblau“ in der Farbe der preußischen Uniformen blüht. Zu gegebenen Anlässen trugen auch Kaiser Wilhelms Anhänger Kornblumen als Knopflochblumen im Revers.

Auch im Palmengarten würdigte man den Kaiser mit Kornblumen-Schmuck; im Gesellschaftshaus wurde 1897 zu Ehren des Kaisers, der in diesem Jahr 100 Jahre alt geworden wäre, ein Festmahl veranstaltet. Der Festsaal im Gesellschaftshaus wurde außer mit Lorbeer und Palmen auch mit Kornblumen geschmückt (BörChers 2012). Das Kornblumenerlebnis der königlich-preußischen Familie kann in Ber-

Abb. 3: Auf mageren Standorten ist bei uns auch die Wiesen-Flockenblume, eine Verwandte der Kornblume, heimisch. Sie ist eine wichtige Futterpflanze für Insekten, z. B. für Grünwidderchen (Adscita spec.)

Abb. 4 (Seite 33): Kornblume aus dem Kräuterbuch des Leonhart Fuchs von 1543. 
lin noch heute nachempfunden werden, denn auf der Siegessäule am großen Stern im Tiergarten ist u.a. auch ein Junge (Wilhelm) mit Kornblumenkranz dargestellt (KRAUSCH 2003).

\section{Kornblume als Emblem}

Nach dem Ersten Weltkrieg wurde die Kornblume als Lieblingsblume der preußischen Königin im Emblem des Bundes Königin Luise aufgegriffen. Die auch als Luisenbund bezeichnete Vereinigung war eine nationalistische und monarchistische Frauenorganisation, die während der Weimarer Republik gegründet und 1934 von den Nationalsozialisten wieder aufgelöst wurde. Bis heute verwenden diverse Vereinigungen die Kornblume als Symbol für Natürlichkeit, Klarheit, Freiheit oder auch Hoffnung. So war es z. B. in der ersten Hälfte des 20. Jahrhunderts die Wandervogelbewegung, die die blaue Blume (gemeint ist hiermit die Kornblume) in ihren Liedern als Inbegriff der Naturverbundenheit pries (BEUCHERT 2004).

„Wir wollen zu Land ausfahren, wohl über die Fluren weit, aufwärts zu den klaren Gipfeln der Einsamkeit. Woll'n lauschen woher der Sturmwind braust, lauschen was hinter den Bergen haust und wie die Welt so weit, und wie die Welt so weit...

... Es blüht im Walde tief drinnen die blaue Blume fein, die Blume zu gewinnen ziehn wir ins Land hinein. Es rauschen die Bäume, es murmelt der Fluss, und wer die blaue Blume finden will, der muss ein Wandervogel sein."

Für Hoffnung und Perspektive steht vermutlich der Name „Kornblume“ eines in Augsburg ansässigen gemeinnützigen Vereins zur Hilfe für Suchtkranke und ihre Angehörigen. Die Kornblume hat es bis zur Nationalblume geschafft. Sie ist offizielle Staatsblume von Estland und wird dort Rukkilill (= Roggenblume) genannt. Man schmückt sich mit ihr zu verschiedenen Anlässen, Mädchen tragen auf Sommerfesten Kornblumenkränze im Haar. Während der Zeit der sowjetischen Besetzung Estlands fand 1969 das 17. estnische Sängerfest

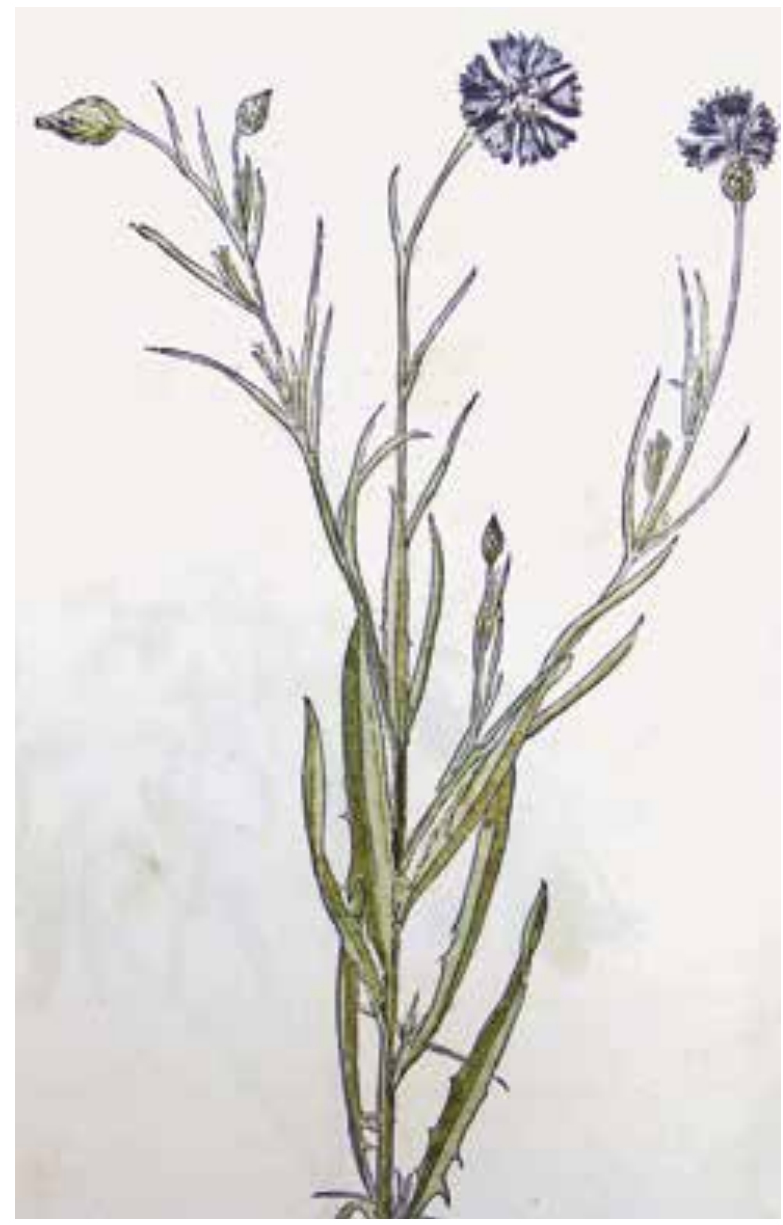

statt. Die Sowjetbehörden betrachteten den Blumenschmuck aus blauen Kornblumen als Symbol gegen den Kommunismus und befahlen, die Blüten rot umzufärben. Nun sollten sie an die roten sozialistischen Nelken erinnern und „politisch korrekt" sein.

\footnotetext{
Literatur

Baumann, H. 1993: Die griechische Pflanzenwelt in Mythos, Kunst und Literatur. - München.

Beuchert, M. 2004: Symbolik der Pflanzen. - Frankfurt a. M.

Börchers, S. 2012: Wo Frankfurts Bürger feiern. Das Gesellschafthaus im Palmengarten. - Frankfurt a. M. Fuchs, L. 1543: New Kreüterbuch. - Reprint 2001, Köln. Krausch, H.-D. 2003. „Kaiserkron und Päonien rot ...“. - München, Hamburg.

Perger, Ritter v., A. 1864: Deutsche Pflanzensagen. Stuttgart, Oehringen.

Schönfelder, I. \& Schönfelder, P. 2001: Der neue Kosmos Heilpflanzenführer. - Stuttgart.
} 